\title{
White Matter Tract Pathology in Pediatric Anoxic Brain Injury from Drowning
}

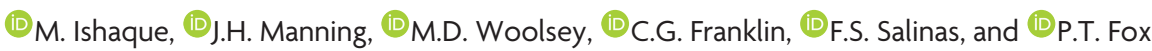
ON EQ

\begin{abstract}
BACKGROUND AND PURPOSE: Although drowning is a leading cause of mortality and morbidity in young children, the neuropathologic consequences have not been fully determined. The purpose of this article was to quantitatively characterize white matter microstructural abnormalities in pediatric anoxic brain injury from nonfatal drowning and investigate the correlation with motor function.
\end{abstract}

MATERIALS AND METHODS: Whole-brain T1-weighted and diffusion-weighted MR imaging datasets were acquired in 11 children with chronic anoxic brain injury and 11 age- and sex-matched neurotypical controls (4-12 years of age). A systematic evaluation form and scoring system were created to assess motor function. Tract-Based Spatial Statistics was used to quantify between-group alterations in the diffusion tensor imaging indices of fractional anisotropy and mean diffusivity and to correlate with per-subject functional motor scores.

RESULTS: Group-wise Tract-Based Spatial Statistics analyses demonstrated reduced fractional anisotropy in the bilateral posterior limbs of the internal capsule and the splenium of the corpus callosum $(P<.001)$. Mean diffusivity was more diffusely increased, affecting the bilateral superior corona radiata, anterior and posterior limbs of the internal capsule, and external capsules $(P<.001)$. Individual-subject fractional anisotropy and mean diffusivity values derived from the ROIs of the bilateral posterior limbs of the internal capsule strongly correlated with motor scores and demonstrated more potent between-group effects than with ROls of the entire corticospinal tract.

CONCLUSIONS: These data particularly implicate the deep white matter, predominantly the posterior limbs of the internal capsule, as targets of damage in pediatric anoxic brain injury with drowning. The substantial involvement of motor-system tracts with relative sparing elsewhere is notable. These results localize white matter pathology and inform future diagnostic and prognostic markers.

ABBREVIATIONS: $A B I=$ anoxic brain injury; $C S T=$ corticospinal tract; $F A=$ fractional anisotropy; $M D=$ mean diffusivity; PLIC = posterior limb of the internal capsule; TBSS $=$ Tract-Based Spatial Statistics

D rowning is a leading global cause of unintentional injury and death and the most important cause in children younger than 4 years of age. ${ }^{1}$ Drowning followed by successful cardiopulmonary resuscitation (nonfatal drowning) is also most important in young children, with an estimated 2 of 3 surviving. ${ }^{2,3}$ In these

Received August 31, 2016; accepted after revision December 5.

From the Research Imaging Institute (M.I., M.D.W., C.G.F., F.S.S., P.T.F.) and Department of Radiological Sciences (M.I., P.T.F.), University of Texas Health Science Center at San Antonio, San Antonio, Texas; Merrill Palmer Skillman Institute (J.H.M.), Wayne State University, Detroit, Michigan; South Texas Veterans Healthcare System (P.T.F.), San Antonio, Texas; and Shenzhen University School of Medicine (P.T.F.), Shenzhen, People's Republic of China.

This work was supported by the National Institutes of Health (R01 MH074457; TL1 TR001119) and the Kronkosky Charitable Foundation. The Conrad Smiles Fund (www.conradsmiles.com) publicized this study and provided funding for travel and logistical support. Miracle Flights for Kids supported airfare costs when possible.

Please address correspondence to Mariam Ishaque, PhD, and Peter T. Fox, MD, Research Imaging Institute, University of Texas Health Science Center at San Antonio, 7703 Floyd Curl Dr, San Antonio, TX 78229; e-mail: Ishaque@livemail.uthscsa.edu; Fox@uthscsa.edu patients, anoxic brain injury (ABI) ensues from the particular dependence of the brain on a continuous oxygen supply, thereby leading to varying levels of neurologic morbidity and, typically, substantial motor dysfunction. ${ }^{1}$

Although the full extent of neuropathologic consequences from $\mathrm{ABI}$ in drowning remains to be established, the resultant injury has been characterized as predominantly affecting gray matter (over white matter), largely on the basis of the metabolic demand profiles of the tissues. ${ }^{4,5}$ In our recent voxel-based morphometric analyses of T1-weighted MR imaging data in children with drowning-related ABI, however, we observed central subcortical tissue loss (in the lenticulostriate arterial distribution) affecting both gray and white matter. ${ }^{6}$ We now aim to specifically assess white

\footnotetext{
- Indicates open access to non-subscribers at www.ajnr.org

$\equiv$ Indicates article with supplemental on-line table.

Indicates article with supplemental on-line photo.

http://dx.doi.org/10.3174/ajnr.A5097
} 
Table 1: Participant information

\begin{tabular}{lcccc}
\hline \multicolumn{1}{c}{ Group } & $\begin{array}{c}\text { Sex } \\
(\mathbf{M} / \mathrm{F})\end{array}$ & $\begin{array}{c}\text { Age at } \\
\text { Injury } \\
(\mathbf{y r}) \text { (Mean) }\end{array}$ & $\begin{array}{c}\text { Age at Scan } \\
\text { (yr) (Mean) }\end{array}$ & $\begin{array}{c}\text { Time since } \\
\text { Injury } \\
(\mathbf{y r}) \text { (Mean) }\end{array}$ \\
\hline All ABI $(n=11)$ & $8 / 3$ & $2.5 \pm 1.1$ & $7.7 \pm 2.9$ & $5.2 \pm 3.1$ \\
All controls $(n=11)$ & $8 / 3$ & - & $7.2 \pm 2.2$ & - \\
Incl. ABI $(n=5)$ & $3 / 2$ & $2.4 \pm 0.8$ & $6.7 \pm 2.8$ & $4.3 \pm 2.2$ \\
Incl. controls $(n=8)$ & $6 / 2$ & - & $6.9 \pm 2.4$ & - \\
\hline
\end{tabular}

Note:-All indicates all participants scanned for our investigation; Incl., participants included in the present study.

matter microstructural integrity in this population by using an imaging technique and analysis method more robust for this purpose.

DTI allows in vivo assessment of white matter microstructure by measuring water diffusion properties. Tract-Based Spatial Statistics (TBSS; http://fsl.fmrib.ox.ac.uk/fsl/fslwiki/TBSS) is an optimized DTI method that mediates group-wise, voxelwise, quantitative white matter analysis. ${ }^{7}$ Here, we used TBSS to measure whole-brain white matter abnormalities and assess motor function correlates in pediatric patients who sustained $\mathrm{ABI}$ from drowning. To our knowledge, this is the first study to quantitatively characterize white matter microstructural damage in drowning.

\section{MATERIALS AND METHODS \\ Participants}

Data were acquired from 22 subjects: 11 children with chronic $\mathrm{ABI}$ from nonfatal drowning and 11 age- and sex-matched neurotypical controls. Inclusion criteria for patients with $\mathrm{ABI}$ were the following: 1) a medically stable state, 2) at least 6 months postinjury, 3) no contraindications to MR imaging, and 4) normal sleep-wake cycles (because children were imaged during sleep). Participant information is shown in Table 1.

All participants' parents provided written consent to the protocol of the study, approved by the institutional review board of the University of Texas Health Science Center at San Antonio. All participants received a stipend.

\section{Image Acquisition}

MR imaging data were obtained on a 3T Tim Trio scanner (Siemens, Erlangen, Germany) with a standard 12-channel head coil as a radiofrequency receiver and the integrated circularly polarized body coil as the radiofrequency transmitter. T1-weighted images were acquired during mildly sedated sleep (1-2 mg/kg of diphenhydramine $\mathrm{HCl}$ ) with the MPRAGE pulse sequence (TR/ $\mathrm{TE}=2200 / 2.72 \mathrm{~ms}$, flip angle $=13^{\circ}, \mathrm{TI}=766 \mathrm{~ms}$, volumes $=$ 208 , and 0.8 -mm isotropic voxel size). A single-shot, single refocusing spin-echo, EPI sequence was used to acquire diffusionweighted data with a spatial resolution of $1.7 \times 1.7 \times 3 \mathrm{~mm}$. The sequence parameters were the following: $\mathrm{TR} / \mathrm{TE}=7800 / 88 \mathrm{~ms}$, FOV $=220 \mathrm{~mm}, 55$ isotropically distributed diffusion-weighted directions, 2 diffusion-weighting values $\left(b=0\right.$ and $\left.700 \mathrm{~s} / \mathrm{mm}^{2}\right)$, and $3 b=0$ (non-diffusion-weighted) images.

\section{DTI Preprocessing and Tract-Based Spatial Statistics}

Diffusion-weighted data were preprocessed by using the FMRIB Diffusion Toolbox (http://fsl.fmrib.ox.ac.uk/fsl/fslwiki/FDT) part of FSL (http://www.fmrib.ox.ac.uk/fsl). ${ }^{8}$ Raw diffusion MR imaging data were corrected for eddy currents and head motion by using the Eddy Current Correction tool. Voxelwise statistical analyses of fractional anisotropy (FA) and mean diffusivity (MD)

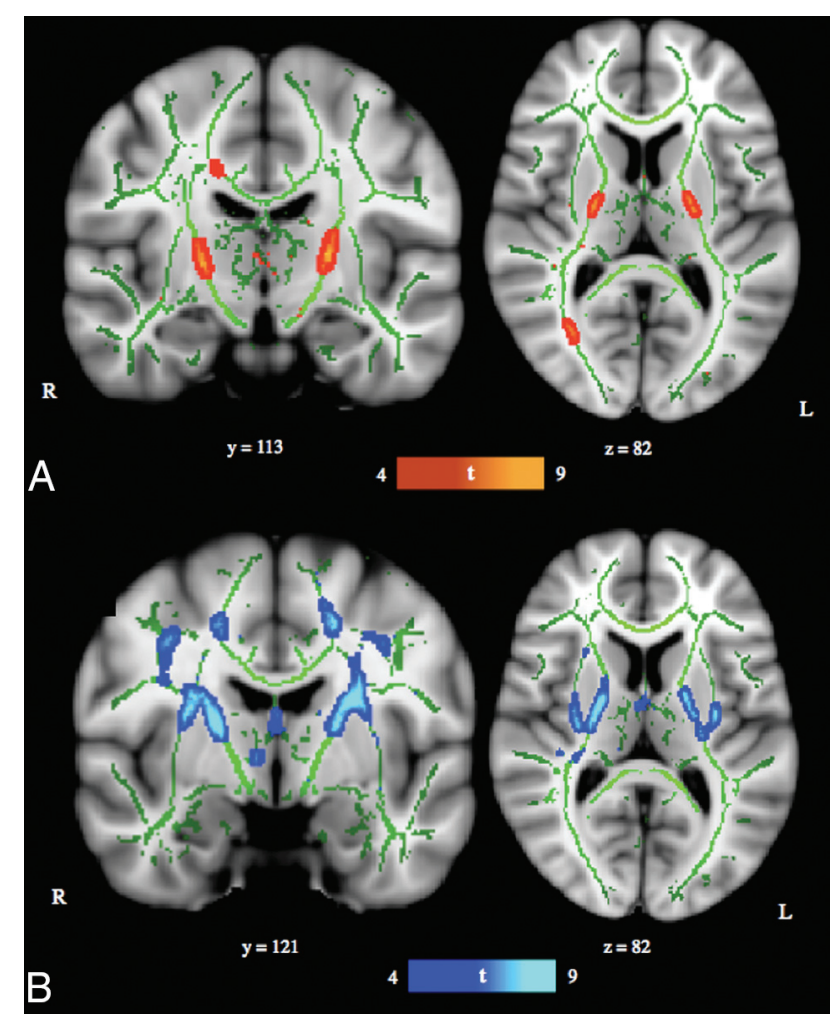

FIG 1. TBSS results. A, TBSS-derived t-map of decreased fractional anisotropy in the anoxic brain injury group relative to the neurotypical control group is shown in red-yellow $(P<.001$, corrected for multiple comparisons). $B$, TBSS-derived $t$-map of increased mean diffusivity in the $A B I$ group relative to the neurotypical control group is shown in blue-light blue ( $P<.001$, corrected for multiple comparisons). Results were thickened with tbss_fill and are overlaid onto the study-specific white matter skeleton (green) and the Montreal Neurological Institute152 template. Section position (given by y or z location) corresponds to Montreal Neurological Institute-152 template space.

data were successively performed by using Tract-Based Spatial Statistics $^{7}$ within FSL. FA/MD images were created by fitting a tensor model to the raw diffusion data with DTIFIT and then brain-extracted with the FSL Brain Extraction Tool (http://fsl. fmrib.ox.ac.uk/fsl/fslwiki/BET). ${ }^{9}$ All subjects' FA/MD data were aligned into a common space (Montreal Neurological Institute) first with linear registration in the FMRIB Linear Image Registration Tool (FLIRT; http://www.fmrib.ox.ac.uk/) ${ }^{10,11}$ external to the TBSS environment, and then by using the FMRIB Nonlinear Registration Tool (FNIRT; http://fsl.fmrib.ox.ac.uk/fsl/fslwiki/ FNIRT). ${ }^{12,13}$ FNIRT uses a b-spline representation of the registration warp field. ${ }^{14}$ The mean FA/MD images were created and thinned to create mean FA/MD skeletons, which represent the centers of all tracts common to the group. Each subject's aligned FA/MD data were projected onto this skeleton, and the resulting data were fed into voxelwise cross-subject statistics. The MELODIC mixture modeling tool (http://fsl.fmrib.ox.ac.uk/fsl/fslwiki/MELODIC) ${ }^{15}$ was applied to raw Tstat images to produce false discovery rate-corrected, thresholded statistical images (Fig 1). For visual purposes, skeletonized results were thickened by using the tbss_fill tool in FSL (http:// fsl.fmrib.ox.ac.uk/fsl/fslwiki/TBSS/UserGuide\#Displaying_ TBSS_Results). Maxima locations were derived from the Johns Hopkins University ICBM-DTI-81 atlas (http://neuro.debian. net/pkgs/fsl-jhu-dti-whitematter-atlas.html). ${ }^{16}$

AJNR Am J Neuroradiol 38:814-19 Apr 2017 www.ajnr.org 

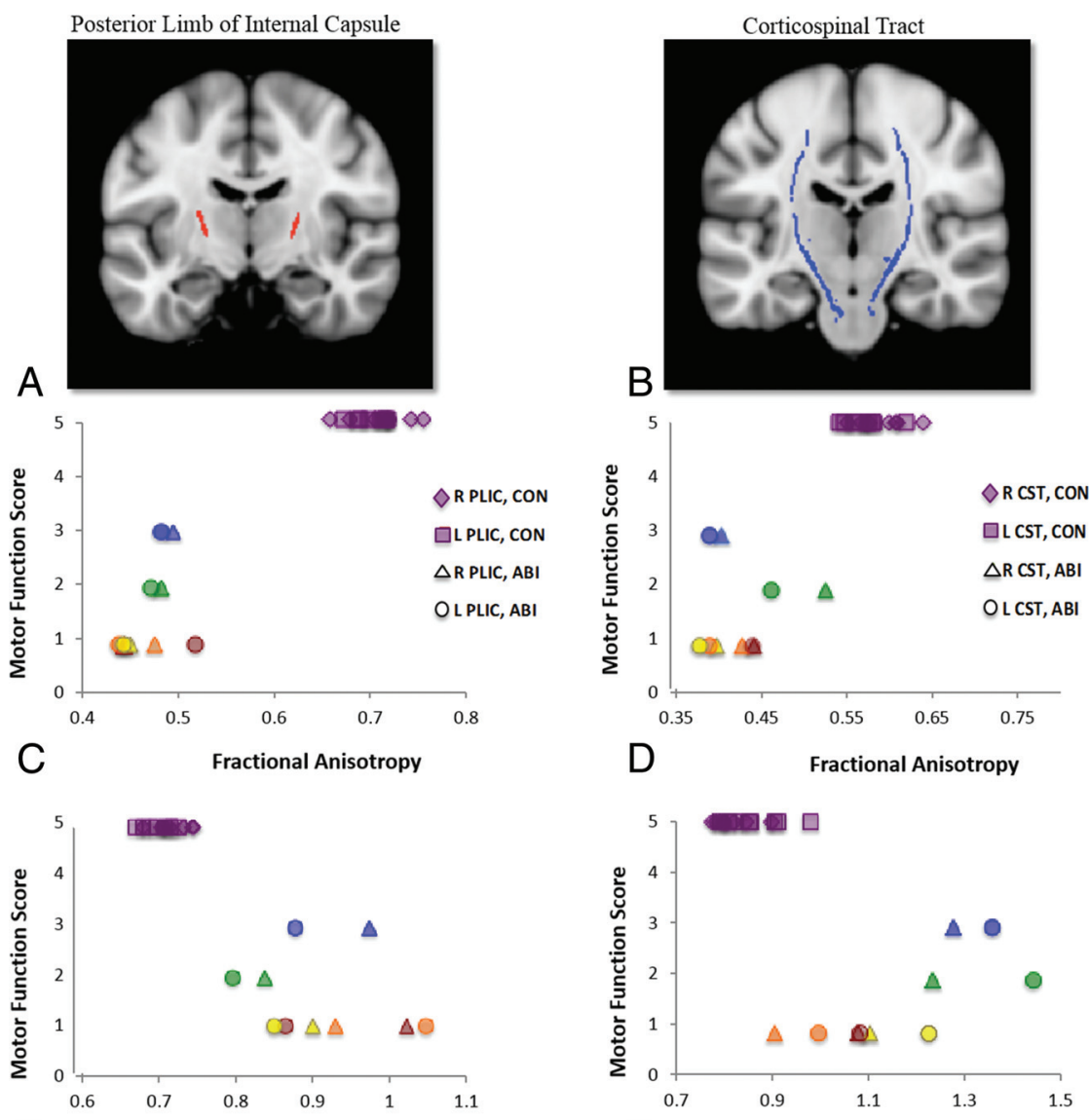

$E$

Mean Diffusivity $\left(x 10^{-3} \mathrm{~mm}^{2} / \mathrm{s}\right)$

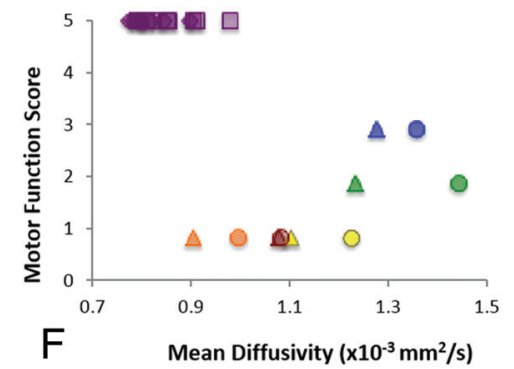

FIG 2. Motor function scores versus diffusion indices. Scatterplots of per-subject motor function scores versus per-subject mean fractional anisotropy and mean diffusivity values from ROI analyses of the right and left posterior limbs of the internal capsule ( $A$, red) and the right and left corticospinal tracts ( $B$, blue) are shown. Motor scores are derived from our functional assessment forms, PLIC ROIs were derived from clusters in our TBSS analysis of FA data, and CST ROIs were derived from the Johns Hopkins University white matter tractography atlas. ABI patient data are color-coded per subject for bilateral PLICs and CSTs: Blue indicates patient A; green, patient B; yellow, patient C; orange, patient D; red, patient E. C, Motor function scores versus FA, right and left PLICs. D, Motor function scores versus FA, right and left CSTs. E, Motor function scores versus MD, right and left PLICs. F, Motor function scores versus MD, right and left CSTs. Analysis of FA data in the bilateral PLICs demonstrates the strongest between-group effects (at the individual subject level), fully differentiating control (CON) and subjects with anoxic brain injury.

Table 2: Motor function correlation with diffusion indices ${ }^{a}$

\begin{tabular}{llclc}
\hline \multirow{2}{*}{ ROI } & \multicolumn{3}{c}{ Spearman $\boldsymbol{\rho}$} \\
\cline { 2 - 5 } & \multicolumn{2}{c}{ FA } & \multicolumn{2}{c}{ MD } \\
\hline Right PLIC & 0.807 & $(P<.001)$ & -0.827 & $(P<.001)$ \\
Left PLIC & 0.814 & $(P<.001)$ & -0.827 & $(P<.001)$ \\
Right CST & 0.845 & $(P<.005)$ & -0.776 & $(P<.005)$ \\
Left CST & 0.807 & $(P<.005)$ & -0.777 & $(P<.005)$ \\
\hline
\end{tabular}

${ }^{a}$ Spearman rank correlation coefficients $(\rho)$ between per-subject motor scores and FA or MD values from ROI analyses of the right and left posterior limbs of the internal capsule $(P<.001)$ and corticospinal tracts $(P<.005)$ are reported.

The final sample consisted of 8 neurotypical and 5 children with $\mathrm{ABI}$ after exclusion of datasets with excessive motion and/or inadequate registration to the standard template. See Table 1 for participant data.

\section{Functional Assessment}

A systematic assessment form with a Likert-type (1-5) scoring system probing motor, sensory, and cognitive aspects of behavior

\section{RESULTS}

was created, with 1 denoting the lowest function and 5 denoting normal function. Data on the functional abilities of the children with ABI were collected through initial assessments by a neurologist (P.T.F.) at the time of imaging and subsequently through extensive interviews with the children's family members, nurses, teachers, therapists, and so forth. In the present study, motor function data were used to assess correlation of behavioral measures with per-subject diffusion imaging parameters (FA and $\mathrm{MD}$ ) in motor tracts. The motor-system assessment, as follows, was largely derived from extant cerebral palsy gross motor function classification systems ${ }^{17}$ : 1) no self-mobility, wheelchair transported; 2) self-mobility in managed situations, wheelchair transported; 3) effective self-mobility otherwise, not walking (wheelchair, rolling, scooting, and so forth); 4) walking with limitations, including hand-held mobility devices (cane or walker); and 5) walking without limitations. See the On-line Table for individual subject measures.

\section{Correlation Analysis}

Two sets of ROI analyses were conducted to study motor tract integrity. The first focused on the right and left posterior limbs of the internal capsule (PLICs), with regions derived from our TBSS analysis of FA data (Fig 2A). The second focused on the right and left corticospinal tracts (CSTs) within the brain, with regions derived from the Johns Hopkins University white matter tractography atlas ${ }^{16}$ and restricted to the white matter skeleton (Fig $2 B$ ). Because the corticospinal tracts carry motor information from the cortex to the spinal cord, they traverse and form a large part of the PLICs. Thus, both sets of ROIs query motor pathway integrity. Individual subjects' mean FA and MD values were extracted from the respective right and left portions of each ROI by using the fslstats tool (https://fsl. fmrib.ox.ac.uk/fsl/fslwiki/Fslutils). Correlations of these values with motor functional scores were measured as Spearman rank correlation coefficients in the $\mathrm{R}$ statistical and computing software environment (http://www.r-project.org). Individual subject motor function scores and DTI indices for the PLICs and CSTs are depicted in Fig 2; correlation coefficients are reported in Table 2.

\section{Voxelwise TBSS Analyses}

Significant regions with decreased FA in the ABI group relative to the neurotypical control group were localized to bilateral PLICs 


\begin{tabular}{llrrr}
\hline \multicolumn{1}{c}{ Anatomic Location } & & \multicolumn{3}{c}{$\begin{array}{c}\text { MNI Coordinates } \\
\text { of Global Maxima }\end{array}$} \\
\cline { 3 - 5 } & Hemisphere & X & Y & Z \\
\hline Fractional anisotropy, control > ABI & & & & \\
PLIC & $\mathrm{L}$ & -22 & -12 & 7 \\
PLIC & $\mathrm{R}$ & 20 & -8 & 8 \\
$\quad$ Corpus callosum, splenium & $\mathrm{R}$ & 20 & -38 & 31 \\
Mean diffusivity, ABI > control & & & & \\
Superior corona radiata & $\mathrm{R}$ & 27 & -8 & 44 \\
Superior corona radiata & $\mathrm{L}$ & -21 & -24 & 43 \\
Internal capsule & $\mathrm{R}$ & 25 & 2 & 20 \\
Internal capsule & $\mathrm{L}$ & -26 & -2 & 22 \\
External capsule & $\mathrm{R}$ & 29 & -6 & 18 \\
External capsule & $\mathrm{L}$ & -32 & -11 & 9 \\
\hline
\end{tabular}

Note:-MNI indicates Montreal Neurological Institute; L, left; R, right.

${ }^{a}$ Location and coordinates of peaks of significant white matter abnormalities in FA and MD are reported $(P<.001$; clusters, $>100 \mathrm{~mm}^{3}$ ). White matter tissue labels derived from the Johns Hopkins University ICBM-DTI-81 atlas. often manifests as decreased directionality of diffusion or decreased fractional anisotropy, and increased water mobility or increased mean diffusivity. ${ }^{18} \mathrm{Al}$ though a single DTI index (ie, FA) can be a sensitive biomarker for neuropathology, the use of multiple diffusion indices and knowledge of the underlying disease mechanism mitigate its relatively low specificity. ${ }^{18,19}$

In pediatric drowning, there is limited knowledge of the extent of brain injury from anoxia, especially in white matter. ABI or hypoxic-ischemic brain injury is most often thought to selectively target gray matter due to its increased metabolic demands and higher and the splenium of the corpus callosum (right hemisphere) $(P<$ .001; Fig $1 A$ and Table 3). Significant regions with increased MD in the $\mathrm{ABI}$ group relative to the neurotypical control group were localized to the bilateral superior corona radiata, posterior and anterior limbs of the internal capsules, and external capsules $(P<$ .001 ; Fig $1 B$ and Table 3$)$. When the statistical threshold was relaxed $(P<.01)$, the output FA and MD differences remained predominately localized to the aforementioned anatomic regions (On-line Figure). There were no significant regions of increased FA or decreased MD in the ABI group relative to the neurotypical control group.

\section{ROI Analyses and Correlations}

With both sets of PLIC and CST ROIs, high correlations were observed with individual subject FA and MD values and motor function (Table 3). Higher FA and lower MD in the bilateral PLICs and CSTs notably correlated with higher motor function scores. The strongest correlation with motor function $(\rho=0.845)$ was measured by using FA values in the right CST. The weakest correlations with motor function were measured by using MD values in the right $(\rho=-0.776)$ and left $(\rho=-0.777)$ CSTs. The test most effective in dissociating patient and control groups was FA analysis of the right and left PLICs, with discrete clusters corresponding to each respective group (Fig $2 C$ ).

\section{DISCUSSION}

White matter microstructural abnormalities in children with ABI from drowning were found to largely implicate deep, central white matter regions. Focal differences in fractional anisotropy were detected in the bilateral PLICs and the splenium of the corpus callosum. Focal differences in mean diffusivity were detected in the bilateral superior corona radiata, internal capsules, and external capsules. Regions of the white matter skeleton demonstrating significant abnormalities in both diffusion metrics were thus within the internal capsules and specifically in the posterior limbs. In individual subjects, FA and MD values from both the PLICs and CSTs highly correlated with corresponding motor function scores, but the PLICs were generally more robust as ROIs.

DTI is a powerful, highly clinically relevant, and readily available method for detecting microscopic changes in tissue architecture. Disruption or loss of white matter structural integrity most concentrations of excitatory neurotransmitter receptors. ${ }^{4,20}$ White matter involvement has been much more variable in most anoxic etiologies. ${ }^{20}$ Despite the important role of structural neuroimaging in the clinical management of children with $\mathrm{ABI}$, its utility in exposing consistent damage across patients and identifying potential prognostic markers has been stunted by heavy reliance on visual inspection (of usually subtle/nonspecific pathology) and a lack of quantitative, group-wise analyses. Diffusion MR imaging is the most sensitive of the structural imaging modalities for detection of ABI. It could especially benefit from more rigorous analysis to better localize injury and ascertain prognostic and therapeutic correlates. ${ }^{20}$

Our previous voxel-based morphometric analyses in this cohort demonstrated gray and white matter loss in children with ABI that was highly convergent on central subcortical regions, chiefly comprising the basal ganglia nuclei and the PLICs and extending to the thalamus, superior corona radiata, and external capsules. ${ }^{6}$ This topography implicates the distribution of the perforating lenticulostriate arteries, an end-arterial system, and thus suggests an important vascular component to the pathophysiology of pediatric drowning.

The present analysis of TBSS in diffusion MR imaging data entirely supports our white matter voxel-based morphometry findings. In children with $\mathrm{ABI}$, the most affected regions-with reduced $\mathrm{FA}$, increased $\mathrm{MD}$, and the highest average $t$-statistic values-were within the bilateral PLICs. This data-driven analysis independently confirms the pathology of the PLICs by using the imaging technique/pulse sequence (DTI) and analytic tool (TBSS) most suited and standardized for the study of white matter structures. ${ }^{7}$ Infarct of the lenticulostriate arteries, from cardiovascular dysfunction and systemic hypotension, thus needs to be seriously considered as a factor in the pathophysiology of pediatric drowning due to the corresponding distribution of tissue damage. Alternatively, however, the white matter tissue loss and microstructural disturbances we have observed could be secondary degeneration to predominant injury in the basal ganglia (ie, gray matter) from the hypoxic-ischemic insult. Primary-versussecondary axonal damage could be differentiated with acute and longitudinal imaging. Primary white matter injury would be appreciated with DWI acutely. Secondary white matter injury would be appreciable after a delay period, likely in the 
subacute phase, following the process of gray matter damage. This distinction is not possible in the present cohort with chronic hypoxic-ischemic brain injury.

Regardless of the causality of the observed tissue-pathology distribution, the involvement of motor-system components is distinctly striking. The basal ganglia nuclei are integral in motorinformation transmission and voluntary movement, and the PLICs largely comprise corticospinal tract fibers carrying motor information from the primary motor cortex to the spinal cord. ${ }^{6,21}$ Most important, the prevalent insult to cerebral motor networks reported here is concordant with motor impairment characteristically observed both in the pediatric drowning patient population and in our patient cohort. The ages of children at highest risk for drowning (1-4 years; 2.4-year average in our cohort) may explain these observations to some extent. The maturation of areas serving motor (and sensory) functions is thought to precede that of brain regions underlying higher cognitive functions. ${ }^{22}$ Thus, younger children (ie, as in this population) may sustain less direct injury to higher order cerebral networks, which remain relatively immature, and greater damage to the more "primitive" and developed motor networks. Nonetheless, the relative sparing of other cerebral networks raises the possibility that these children retain substantial cognitive, perceptual, and emotional capabilities and that they are unable to effectively convey this functional integrity due to motor-system damage. Further examination of this hypothesis with functional neuroimaging methods is strongly indicated.

In addition to localizing the most consistent pathology in drowning-related pediatric $\mathrm{ABI}$ and characterizing the functional correlates group-wise, we report extremely high correlations of imaging metrics with motor function at the individual-subject level. Furthermore, with FA and MD values from right and left PLIC ROIs (derived from FA TBSS analysis) and subjects' motor function scores, complete dissociation of subjects in the patient and control groups was possible. This was most effectively conducted in FA data (Fig 2). Evaluation of FA and/or MD values by using PLIC ROIs may help prognosticate and monitor therapeutic effects in children recovering from drowning. This would be especially powerful because each subject could serve as his or her own baseline reference, and longitudinal changes in white matter microstructure could be evaluated for response with time and to interventions. In a perinatal anoxia study, TBSS and diffusion metrics in internal capsule ROIs were indeed able to detect the therapeutic efficacy of hypothermia in infants with neonatal encephalopathy. ${ }^{23}$ In another study of patients with chronic stroke, FA measurements in the corticospinal tracts correlated with the potential for motor functional recovery. ${ }^{24}$ Such analyses would also be feasible and potentially quite useful in the patient population herein described.

To assess the specificity of injury in this cohort, ROI analyses were also implemented in the corticospinal tracts. CST ROIs were derived from a white matter atlas and subjected to the same analysis as the PLIC ROIs. Although high correlations with motor function scores were also measured by using the CSTs, they were not as effective in delineating patient and control subjects. This finding is not surprising considering the origin of the ROIs: The PLIC regions used in the correlation analysis represent the most significant between-group differences in FA (and MD), while the
CST regions encompass proximal and distal areas as well. The presence (or relative greater quantity) of crossing fibers in the CST ROIs may also, in part, contribute to this disparity. Injury in pediatric $\mathrm{ABI}$ from drowning, nevertheless, does appear to be most specific to the PLICs.

Several future directions are important. Probabilistic diffusion tractography studies are indicated and quite well-informed by the findings reported here. Probabilistic tractography can be implemented at the per-subject level to obtain connectivity indices reflecting intact fiber organization. ${ }^{25}$ Although the typical method entails seed-to-target connectivity measurements across a tract (ie, the entire CST), our results suggest that it would be more powerful to measure connectivity across the PLICs. Our findings also prompt future clinical applications in targeted MR spectroscopy and endovascular therapies to the lenticulostriate arteries via the middle and/or anterior cerebral arteries, as described in Ishaque et al. ${ }^{6}$ Further testing is warranted via treatment trails in an anoxic brain injury model in nonhuman primates.

This study has limitations. Diffusion-weighted MR imaging is known to be extremely sensitive to subject motion, eddy currents, and magnetic field inhomogeneities. ${ }^{18}$ Additionally, we were rather stringent with data inclusion to ensure quantitative analysis of high-quality data. This accordingly resulted in the exclusion of 3 control and $6 \mathrm{ABI}$ datasets, either due to excessive motion in the first instance or inadequate registration within the TBSS analysis thereafter. Thus, we acknowledge that our findings are from a small sample size. Diffusion MR imaging data acquisition would likely benefit from increased sedation of pediatric subjects. We emphasize, however, that substantial (and focal) between-group differences were nevertheless observed at quite high statistical significance levels. We are thereby confident that our findings reflect underlying disease-specific white matter pathology. Nevertheless, we interpret our results as specific to pediatric $\mathrm{ABI}$ from drowning and recognize that they may not generalize to other anoxic etiologies or age groups. For clinical purposes, the group-wise study design we use is also a limitation. An alternative for future exploration would be per-subject probabilistic tractography, as discussed above. The most promising method as we see it would involve tractography of the PLICs.

\section{CONCLUSIONS}

This study reports the first quantitative, whole-brain, voxelwise characterization of white matter microstructural changes in pediatric ABI from drowning. TBSS analysis of FA and MD indices demonstrated substantial disruption of the deep, central white matter, predominantly implicating motor tracts; these results largely correlated with motor functional abilities at the individual-subject level. Other white matter tracts were observed to be relatively preserved. These findings support our recent gray and white matter voxel-based morphometry analyses that localized structural pathology to the basal ganglia and internal capsules and, together, suggest primary motor-system damage in this population. The prospect of motor nuclei/motor pathway injury masking relatively intact cognitive, perceptual, and emotional abilities in children with $\mathrm{ABI}$ from drowning must be further investigated. Additionally, our reported results motivate important diagnostic, prognostic, and therapeutic considerations. 


\section{ACKNOWLEDGMENTS}

The authors thank all families for their time and participation in this study and Dr Christian F. Beckmann, PhD (University of Oxford, Donders Institute), for his data-analysis suggestions.

Disclosures: Mariam Ishaque—RELATED: Grant: National Institutes of Health. * Crystal G. Franklin—RELATED: Grant: National Institutes of Health. * Felipe S. SalinasUNRELATED: Grants/Grants Pending: US Department of Defense, Comments: I received a percentage of salary support for my work in the Consortium to Alleviate Post-Traumatic Stress Disorder (W81XWH-08-2-0112); Other: National Institutes of Health/National Institute of Neurological Disorders and Stroke, Comments: I also received a percentage of salary support for my work in epilepsy research on a National Institutes of Health-funded R21 (IR21NS084198-01A1).* Peter T. FoxRELATED: Grant: Kronkosky Charitable Foundation, Comments: The grant funded the study, including acquiring scans and paying a salary stipend for the first author.* *Money paid to the institution.

\section{REFERENCES}

1. Topjian AA, Berg RA, Bierens JJ, et al. Brain resuscitation in the drowning victim. Neurocrit Care 2012;17:441-67 CrossRef Medline

2. Borse N, Sleet DA. CDC childhood injury report: patterns of unintentional injuries among 0- to 19-year olds in the United States, 2000-2006. Fam Community Health 2009;32:189 CrossRef Medline

3. Kriel RL, Krach LE, Luxenberg MG, et al. Outcome of severe anoxic/ ischemic brain injury in children. Pediatr Neurol 1994;10:207-12 CrossRef Medline

4. Huang BY, Castillo M. Hypoxic-ischemic brain injury: imaging findings from birth to adulthood. Radiographics 2008;28:417-39; quiz 617 CrossRef Medline

5. Hegde AN, Mohan S, Lath N, et al. Differential diagnosis for bilateral abnormalities of the basal ganglia and thalamus. Radiographics 2011;31:5-30 CrossRef Medline

6. Ishaque M, Manning JH, Woolsey MD, et al. Lenticulostriate arterial distribution pathology may underlie pediatric anoxic brain injury in drowning. Neuroimage Clin 2016;11:167-72 CrossRef Medline

7. Smith SM, Jenkinson M, Johansen-Berg H, et al. Tract-based spatial statistics: voxelwise analysis of multi-subject diffusion data. Neuroimage 2006;31:1487-505 CrossRef Medline

8. Smith SM, Jenkinson M, Woolrich MW, et al. Advances in functional and structural MR image analysis and implementation as FSL. Neuroimage 2004;23(suppl 1):S208-19 CrossRef Medline

9. Smith SM. Fast robust automated brain extraction. Hum Brain Mapp 2002;17:143-55 CrossRef Medline

10. Jenkinson M, Smith S. A global optimisation method for robust affine registration of brain images. Med Image Anal 2001;5:143-56 CrossRef Medline
11. Jenkinson $M$, Bannister $P$, Brady $M$, et al. Improved optimization for the robust and accurate linear registration and motion correction of brain images. Neuroimage 2002;17:825-41 CrossRef Medline

12. Andersson J, Jenkinson M, Smith S. Non-linear optimization: FMRIB technical report TR07JA1. Oxford, United Kingdom: FMRIB Centre; 2007. http://www.fmrib.ox.ac.uk/datasets/techrep/tr07ja1/tr07ja1. pdf. Accessed February 11, 2016

13. Andersson J, Jenkinson M, Smith S. Non-linear registration, aka Spatial normalization: FMRIB technical report TR07JA2. Oxford, United Kingdom: FMRIB Centre; 2007. http://www.fmrib.ox.ac.uk/datasets/ techrep/tr07ja2/tr07ja2.pdf. Accessed February 11, 2016

14. Rueckert D, Sonoda LI, Hayes C, et al. Nonrigid registration using free-form deformations: application to breast MR images. IEEE Trans Med Imaging 1999;18:712-21 CrossRef Medline

15. Woolrich MW, Behrens TEJ, Beckmann CF, et al. Mixture models with adaptive spatial regularization for segmentation with an application to FMRI data. IEEE Trans Med Imaging 2005;24:1-11 CrossRef Medline

16. Mori S, Crain BJ. MRI Atlas of Human White Matter. Amsterdam: Elsevier; 2005

17. Palisano R, Rosenbaum P, Walter S, et al. Development and reliability of a system to classify gross motor function in children with cerebral palsy. Dev Med Child Neurol 1997;39:214-23 Medline

18. Alexander AL, Lee JE, Lazar M, et al. Diffusion tensor imaging of the brain. Neurotherapeutics 2007;4:316-29 CrossRef Medline

19. Yamada K, Sakai K, Akazawa K, et al. MR tractography: a review of its clinical applications. Magn Reson Med Sci 2009;8:165-74 CrossRef Medline

20. Rabinstein AA, Resnick SJ. Practical Neuroimaging in Stroke: A CaseBased Approach. Philadelphia: Saunders/Elsevier; 2009

21. Anderson JC, Costantino MM, Stratford T. Basal ganglia: anatomy, pathology, and imaging characteristics. Curr Probl Diagn Radiol 2004;33:28-41 CrossRef Medline

22. de Bie HM, Boersma M, Adriaanse $S$, et al. Resting-state networks in awake five- to eight-year old children. Hum Brain Mapp 2012;33: 1189-201 CrossRef Medline

23. Porter EJ, Counsell SJ, Edwards AD, et al. Tract-based spatial statistics of magnetic resonance images to assess disease and treatment effects in perinatal asphyxial encephalopathy. Pediatr Res 2010;68: 205-09 CrossRef Medline

24. Stinear CM, Barber PA, Smale PR, et al. Functional potential in chronic stroke patients depends on corticospinal tract integrity. Brain 2007;130:170-80 Medline

25. Behrens TE, Berg HJ, Jbabdi S, et al. Probabilistic diffusion tractography with multiple fibre orientations: what can we gain? Neuroimage 2007;34:144-55 CrossRef Medline 